\title{
Iron deficiency anaemia in pregnancy and its prevention; paradigm changes over three decades
}

\author{
Malik Goonewardene \\ Emeritus Professor of Obstetrics and Gynaecology, University of Ruhuna, Galle, Sri Lanka. \\ Correspondence: Prof. Malik Goonewardene \\ e-mail: malikg.obsgyn@med.sab.ac.lk/malikg@eureka.lk \\ https://orcid.org/0000-0002-7899-7117
}

THE GALLE MEDICAL ASSOCIATION ORATION - 2020

\begin{abstract}
In Sri Lanka, the prevalence of anaemia in pregnancy was estimated to be approximately $29 \%$ in 2001 but is estimated to have significantly reduced to be $<20 \%$, at least in certain areas, during the last decade. Among its many causes, nutritional iron deficiency anaemia (IDA) is the commonest, but establishing a definitive diagnosis is difficult. Strategies adopted globally, as well as in Sri Lanka, to prevent IDA in pregnancy, have changed significantly during the last three decades.

A comprehensive literature review was carried out and inter alia three relevant World Health Organization Guidelines, in which the author was a member of the guideline development group and six other publications of the author, on the topic of iron deficiency (ID) and IDA in pregnancy are included in this review. Ten studies on the detection and prevention of ID and IDA in pregnancy, carried out from 1990 to 2017 at the Academic Obstetrics and Gynaecology Unit (AOGU) of the Teaching Hospital Mahamodera, Galle (THMG) are described. The results and conclusions of these studies were: poor compliance and unsatisfactory methods of ingestion of the antenatal oral iron supplement could have contributed to the high rates of ID and IDA at term during the period 1990 to 1992; haematological indices during pregnancy need to be interpreted with caution; although the agreement between haematological indices obtained from different laboratories in Galle in 2000 was unsatisfactory, better agreement was observed in 2015; the rates of ID and IDA in pregnant women presenting for antenatal care to the AOGU of the THMG have significantly decreased from approximately $69 \%$ and $44 \%$ respectively in 1990 to approximately $37 \%$ and $17 \%$ respectively in 2015 . Therefore, weekly antenatal oral iron supplements should be adequate to improve birth outcomes in the non-anaemic women attending this clinic.
\end{abstract}

Key words: Anaemia, antenatal oral iron supplementation, iron deficiency, pregnancy

\section{Introduction}

Anaemia in pregnancy, defined as a haemoglobin concentration $(\mathrm{Hb})<110 \mathrm{~g} / \mathrm{L}$, is a significant public health problem globally (1). Its prevalence in Sri Lanka was estimated to be approximately $29 \%$ in 2001(2) and 34\% in 2007 (3). However in 2009, using a small sample of 228 pregnant women, the prevalence of anaemia during pregnancy was estimated to be approximately $17 \%$ in Sri Lanka, ranging from approximately $7 \%$ in Kurunegala to $29 \%$ in the Colombo Municipality (4). Furthermore, regional studies on the prevalence of anaemia in pregnant women have reported rates of $8.2 \%$ to $14.4 \%$, during the period 1999 to 2012 (5-7), suggesting that the national rate of anaemia in pregnancy could be less than $20 \%$. Several factors affecting $\mathrm{Hb}$ and iron status in pregnancy lead to 
difficulties in establishing a definitive diagnosis (8-12). Furthermore, a healthy woman could progress to a state of latent iron deficiency (ID), then to ID with no anaemia and finally to a clinical iron deficiency anaemia (IDA). Therefore, the prevalence of ID is always greater and could be 2 - 2.5 times the prevalence of IDA $(8,11,12)$. Anaemia in pregnancy, which could be due to multiple factors of which nutritional IDA is the commonest, is associated with increased maternal and perinatal morbidity and mortality, and longterm adverse effects in the new born $(8,9,11-13)$. Although routine daily antenatal oral iron supplementation programmes have been implemented in low income countries for several decades, their effectiveness in preventing IDA at term has been shown to be suboptimal, and its safety, especially in areas where Malaria is prevalent, has also been questioned (14). Although Sri Lanka has been declared "Malaria Free" by the World Health Organization (WHO) in 2016 (15), routine daily antenatal oral iron supplementation would probably be beneficial only in pregnant women with ID $(16,17)$. During the last three decades, as a result of a better understanding of the mechanisms of absorption of oral iron supplements from the gut, there has been a great interest in the role of intermittent iron supplements rather than daily supplements (18-20). The WHO has recommended that iron and folate supplements should be given to all menstruating adult women and adolescents, adjusting the dose and frequency of administration according to the prevalence of anaemia in the particular community $(21,22)$. Consequent to this, a few years ago, a weekly oral iron supplementation programme for four weeks, has been commenced in Lankan schools for grade seven and grade 10 students. It has also been recommended that, in communities where the prevalence of anaemia in pregnancy is $<20 \%$, intermittent (e.g. weekly) antenatal oral iron supplementation should be administered for non anaemic pregnant women (23). Although such a programme could be appropriate at least in certain areas of Sri Lanka, no such programme has been implemented in any area in Sri Lanka up to date.

The aim of this review is to describe the difficulties in establishing a diagnosis of IDA and ID in pregnancy, and the significant changes in the rates of IDA and ID in pregnancy as well as the strategies adopted to prevent IDA and ID in pregnancy, during the period from 1990 to 2017, among pregnant women presenting for antenatal care to the Academic Obstetrics and Gynaecology Unit (AOGU) of the Teaching Hospital Mahamodera, Galle ( THMG).

\section{Methods}

A comprehensive literature review was carried out and inter alia three relevant World Health Organization Guidelines, in which the author was a member of the guideline development group and six other publications of the author, on the topic of ID and IDA in pregnancy, are included in this review. Ten studies on the detection and prevention of ID and IDA in pregnancy, carried out from 1990 to 2017 at the AOGU of the THMG are described. The objectives of these were to: describe the rates of ID and IDA in women presenting for antenatal care in 1990 and compare with the same in 2015 (24.25); describe the effectiveness of the antenatal oral iron supplementation programme in 1992 (26); measure the agreement between haematological indices obtained by different laboratories in 2000 (27) and compare with the same in 2015 (28); assess the validity of commonly used haematological indices (29); describe the effectiveness of intermittent antenatal oral iron supplementation between 1994 to 1996 (30-32) and compare it with the same in $2015 / 2016(33)$.

\section{Studies on the detection and prevention of iron deficiency and iron deficiency anaemia in pregnancy, carried out from 1990 to 2017 at the Academic Obstetrics and Gynaecology Unit of the Teaching Hospital Mahamodera, Galle}

Prior to conducting these studies, ethical approval was obtained from the Ethical Review Committee of the Faculty of Medicine, University of Ruhuna, administrative approval was obtained from the respective Directors of the THMG during the times of the studies, and the randomized control trial (RCT) carried out in 2015/2016 was registered in the Sri Lanka Clinical Trials Registry, which was established in 2006. Informed written consent was obtained from all the women who participated in these studies. 


\section{Study 1 (24)}

A cross sectional analytical study was carried out in 236 consecutive pregnant women during a two months' period from January 1990, using the cyanmethhaemoglobin method, haematocrit tubes and immunoradioemetric assay technique for the measurement of their $\mathrm{Hb}$, hematocrit (Hct) and serum ferritin (SF) respectively. The rates of anaemia and ID were $56 \%$ and $69 \%$ respectively, and $13 \%$ of women had ID although they were not anaemic. The $\mathrm{Hb}$ had a low sensitivity (63\%) and a poor negative predictive value $(41 \%)$ in the detection of ID.

\section{Study $2(25)$}

A cross sectional analytical study was carried out in 350 consecutive pregnant women with gestations between 12 to 20 weeks, presenting from November 2014 to January 2015. Their Hb and Hct were measured by the flow-cytometry and hydrodynamic focusing methods and their SF was measured by the electro-chemiluminescence method. The best cut off level of SF which was associated with anaemia, obtained from a Receiver Operator Characteristics (ROC) curve, was SF $<30 \mu \mathrm{g} / \mathrm{L}$ (area under the ROC curve $=0.77,95 \%$ CI 0.72 to 0.81 ), and it had a sensitivity of $78.3 \%$ $(95 \%$ CI 65.8 - 87.9) and a specificity of $74 \%$ (95\% CI 68.6 - 79.0) in detecting anaemia. Using this $\mathrm{SF}<30 \mu \mathrm{g} / \mathrm{L}$ cut off, $36.9 \%$ of subjects had ID. The rate of anaemia was $16.6 \%$.

\section{Study 3 (26)}

A prospective cohort study was carried out in 88 consecutive pregnant women with gestations between 14 to 24 weeks, during a two months' period commencing 15 May 1992. Their $\mathrm{Hb}$ and SF were measured, using the same methods as in study 1 , before and after varying durations of antenatal, daily, oral supplements which consisted of a tablet containing ferrous sulphate $200 \mathrm{mg}$ (elemental iron $60 \mathrm{mg}$ ) with folate $25 \mu \mathrm{g}$, and another tablet containing calcium lactate pentahydrate $300 \mathrm{mg}$. Women who had an initial $\mathrm{Hb}<10 \mathrm{~g} / \mathrm{dl}$ and $\mathrm{SF}<$ $10 \mathrm{ng} / \mathrm{ml}$ had a marked increase of $\mathrm{Hb}$ and $\mathrm{SF}$ respectively after supplementation $(p<0.001)$, and women who had supplementation for $>12$ weeks had a significant increase of SF $(p<0.05)$. However, there was no significant change in the mean $\mathrm{Hb}$ $(10.9 \mathrm{~g} / \mathrm{dl}, \mathrm{sd} 1.7)$ and mean SF $(23.9 \mathrm{ng} / \mathrm{ml}, \mathrm{sd} 23.5)$ of the 88 women. All the women had taken the iron folate supplement and the calcium supplement together, 87 had taken the supplements after a meal of unpolished rice and vegetables, and $16 \%$ with a cup of tea. Only $58 \%$ had taken the supplements correctly, as advised. Therefore, it was concluded that the compliance and method of intake of the supplements should be improved to enhance the effectiveness of the supplementation programme.

\section{Study 4 (27)}

A cross sectional analytical study was carried on two cohorts of pregnant women presenting for antenatal care in 1990; one in the second trimester $\mathrm{T}^{2}$ (Group A, $\mathrm{n}=156$ ) and the other in the third trimester $-\mathrm{T}^{3}$ of pregnancy (Group B, $\mathrm{n}=47$ ). In Group $\mathrm{A}$, the $\mathrm{Hb}$ and Hct were measured in the same blood sample at the Karapitiya Medical Faculty Laboratory (KMFL) using the cyanometh-haemoglobin method and haematocrit tubes respectively and the Kyoto Medical Laboratory Galle (KMLG) using an automated haematology analyser. In Group B, from the same blood sample, in addition to the $\mathrm{Hb}$ and Hct measurements carried out in KMFL and KMLG another measurement of $\mathrm{Hb}$, using the cyanomethhaemoglobin method, was carried out at the Karapitiya Teaching Hospital Laboratory. In $\mathrm{T}^{2}$, the mean $\mathrm{Hb}$ reported from KMFL was markedly less than the mean $\mathrm{Hb}$ reported from KMLG ( $8.3 \mathrm{~g} / \mathrm{dl}$ sd 1.7 vs $11.4 \mathrm{~g} / \mathrm{dl}$ sd $1.2, p<0.001)$. There were no clinically significant differences in the mean Hct reported from the two laboratories. In $\mathrm{T}^{3}$ there were no significant differences in the mean $\mathrm{Hb}$ reported from the three laboratories. Although there was slight agreement between the reports of three laboratories (Kappa 0.28, $p<0.02$ ) with modest agreement between the reports from the KMFL and KMLG (Kappa 0.62, $p<0.001$ ), individual differences between the reports were clearly seen when the data were plotted graphically. As the $\mathrm{Hb}$ measured by three different laboratories differed significantly from each other, interpreting a single $\mathrm{Hb}$ measurement with caution, and the use of the Hct in addition to the $\mathrm{Hb}$ was recommended for the diagnosis of anaemia. 


\section{Study $5(28)$}

A cross sectional analytical study was carried on 350 consecutive pregnant women, with gestations between 12 to 20 weeks from November 2014 to January 2015. Their $\mathrm{Hb}$ and Hct were measured, in the same blood sample, by flow-cytometry and hydro-dynamic focusing methods at the Durdans Hospital Laboratory, Galle and the colorimetric method at the laboratory of the THMG. No significant differences were seen between the mean $\mathrm{Hb}$ values and between the mean Hct values obtained from the two laboratories. Strong, positive correlations were seen between the $\mathrm{Hb}$ values as well as between the haematocrit values obtained from the two laboratories $(\mathrm{r}=0.86, p<0.001$ and $\mathrm{r}=0.83, p<0.001$ respectively). The limits of agreement and the clinical limits of indifference between the $\mathrm{Hb}$ as well as between the hematocrit values obtained from the two laboratories were satisfactory but individual differences of $>10 \%$ were seen in $6.6 \%$ of results

\section{Study $6(29)$}

In 1990, another cross sectional analytical study was carried on the same two cohorts of pregnant women of Study 4. Haematological indices were measured by an automated hematology analyser and the SF was measured by immunoradiometric assay. For the diagnosis of ID, a Sf $<12 \mathrm{ng} / \mathrm{L}$ was used. Using the accepted cut off values of each haematological index for the diagnosis of anaemia, the validity of detection of ID by each haematological index was measured. The Hct, mean corpuscular volume, and the mean corpuscular haemoglobin concentration (MCHC) had a high specificity (96\%-100\%) but very low sensitivity (10\%-38\%). Only the mean corpuscular haemoglobin had a high sensitivity $\left(92 \%\right.$ in $\mathrm{T}^{2}$ and $86 \%$ in $\left.\mathrm{T}^{3}\right)$, but it had a low specificity $(21 \%$ $24 \%)$. The MCHC had the best accuracy $(71 \%$ in $\mathrm{T}^{3}$ ) but its accuracy in $\mathrm{T}^{2}$ was only $64 \%$. Only the MCHC showed a moderate agreement with the SF (Kappa 0.41, $p<0.001$ ). Therefore, it was recommended that multiple haematological indices should be evaluated before diagnosing ID and deciding on subsequent supplementation or treatment of ID during pregnancy.

\section{Study $7(30)$}

In 1994, a prospective cohort study was carried on 56 consecutive pregnant women with gestations between 13 to 26 weeks. Their $\mathrm{Hb}$ and SF were measured, using the same methods as in studies 1 and 3 , before and after the intake of an oral multi micronutrient capsule, which contained ferrous fumarate $350 \mathrm{mg}$ (elemental iron $117 \mathrm{mg}$ ), folate $1.5 \mathrm{mg}$, cyanocobalamine $15 \mu \mathrm{g}$, calcium carbonate $200 \mathrm{mg}$, cholecalciferol $400 \mathrm{iu}$, and ascorbic acid $75 \mathrm{mg}$. The women were advised to take the capsule with water, once a week, at 11.00 a.m. (approximately one hour before lunch), for 12 weeks. Mebendazole $100 \mathrm{mg}$ twice daily for three days was given prior to commencement of the supplementation. There was $100 \%$ compliance and no side effects were reported. There were no significant changes in the mean $\mathrm{Hb}$ and Hct, in the total study sample, after supplementation. However, there was a significant reduction in the mean $\mathrm{Hb}$ by $1.3 \mathrm{~g} / \mathrm{dl}$ (sed $0.44, p<0.01$ ), in the 13 women who had $\mathrm{Hb}$ of $\geq 11 \mathrm{~g} / \mathrm{dl}$. Furthermore, the number of women with $\mathrm{Hb}<11 \mathrm{~g} / \mathrm{dl}$ and $\mathrm{SF}<12$ $\mathrm{ng} / \mathrm{ml}$ increased from 14/56 before supplementation to 24/56 after supplementation $(p<0.05)$. Therefore, this weekly regimen was inadequate to meet the increased requirements of iron during pregnancy.

\section{Study $8(31)$}

In 1994, a prospective cohort study was carried on 77 consecutive pregnant women with gestations between 14 - 26 weeks. The method adopted was the same as in Study 7, with one important difference. The women were advised to take the supplement every Monday, Wednesday and Friday for 12 weeks. There was $100 \%$ compliance and no side effects were reported. The mean $\mathrm{Hb}$ increased and the number of women with $\mathrm{Hb}<8 \mathrm{~g} / \mathrm{dl}$ and $\mathrm{SF}<12 \mathrm{ng} / \mathrm{ml}$ decreased after supplementation, especially in the 27 women who had initial $\mathrm{Hb}<11$ $\mathrm{g} / \mathrm{dl}$ and $\mathrm{SF}<12 \mathrm{ng} / \mathrm{ml} . \quad(p<0.01)$. However, the mean SF decreased by $14.1 \mathrm{ng} / \mathrm{ml}(\operatorname{sed} 4.9, p<0.05)$ in the 50 women who had an initial SF $>12 \mathrm{ng} / \mathrm{ml}$ and the mean Hct decreased by $2 \%(\operatorname{sed} 0.7, p<0.01)$ in the total study sample. Therefore, although this thrice weekly regimen was beneficial in women with moderate to severe ID, it was inadequate to 
meet the increased requirements of iron during pregnancy in women with borderline or mild iron deficiency.

\section{Study $9(32)$}

In 1995, a randomized controlled trial (RCT) was carried out on 92 consecutive pregnant women with gestations between 14 to 24 weeks. Their Hb, Hct and SF were measured, using the same methods as in studies 1and 3, before and after supplementation. They were treated with mebendazole $100 \mathrm{mg}$ twice a day for three days, and randomly allocated to receive weekly $(\mathrm{n}=26)$, three times a week $(\mathrm{n}=35)$ and daily $(\mathrm{n}=31)$, an oral micro nutrient capsule which was the same as in studies 7 and 8 . The women were advised to take the capsule with water daily, weekly or every Monday, Wednesday and Friday, at 11.00 a.m. (approximately one hour before lunch). Compliance was checked by keeping a record of the number of capsules administered to each participant and reviewing each participant at four weekly intervals. The compliance was very good and no serious side effects were reported. There were reductions in the numbers of women with anaemia in all three groups. However, the number of women with $\mathrm{SF}<12 \mathrm{ng} / \mathrm{ml}$ increased in the weekly supplementation group while they decreased in the daily supplementation group $(p<0.001)$. There were very high risks of developing ID in the weekly and thrice weekly supplementation groups (OR 18, 95\% CI 2.8 - 115.5, $p=0.002$, and OR 10, 95\% CI 1.6 - 64.8, $p<0.015$, respectively). There was an increased risk of developing anaemia in the weekly supplementation groups (OR 15, 95\% CI $14-165.6, p=0.027$ ). Therefore, intermittent regimens were considered to be inadequate and continuation of the daily regimen was recommended.

\section{Study 10 (33)}

During the period December 2014 to April 2015, a RCT was carried out on 292 consecutive, nonanaemic pregnant women with gestations between 14 to 22 weeks, who presented for antenatal care, and who had been treated with mebendazole $100 \mathrm{mg}$ twice a day for three days. They were randomly allocated to receive $120 \mathrm{mg}$ elemental iron, $3 \mathrm{mg}$ folic acid and $100 \mathrm{mg}$ vitamin C weekly $(\mathrm{n}=149)$ or $60 \mathrm{mg}$ elemental iron, $1 \mathrm{mg}$ folic acid and $100 \mathrm{mg}$ vitamin $C$ daily $(n=143)$. All were assessed for side effects at four weekly intervals and their $\mathrm{Hb}$, Hct and SF were measured at 32 to 36 weeks gestation, with the same methods used in Study 2. Only 106 participants in each group completed the study. Between the two groups there were no significant differences in the mean duration of supplementation, the post supplementation mean $\mathrm{Hb}$, Hct and SF, and the risks of developing anaemia, ID or high $\mathrm{Hb}$ levels, by an intension to treat analysis as well as in those who completed the trial. Side effects were significantly greater in the daily supplementation group compared to the weekly supplementation group. Therefore, in non anaemic pregnant women presenting for antenatal care, a weekly regimen was an effective alternative to a daily regimen for antenatal oral iron and folate supplementation, in preventing anaemia and ID in the third trimester.

\section{Conclusions}

Haematological indices during pregnancy need to be interpreted with caution, taking into consideration several factors. Poor compliance and unsatisfactory methods of ingestion of the daily antenatal oral iron supplement could have contributed to the high rates of anaemia and ID at term during the period 1990 to 1992. Although the agreement between haematological indices obtained from different laboratories in Galle were unsatisfactory in 2000, better agreement was observed in 2015. In pregnant women presenting for antenatal care to the AOGU of THMG, the rates of anaemia and ID have markedly decreased from approximately $56 \%$ and $69 \%$ respectively in 1990 to approximately $17 \%$ and $37 \%$ respectively in 2015 . As the rate of IDA is $<20 \%$, weekly antenatal oral iron supplements should be adequate to improve birth outcomes in the non-anaemic women attending this clinic. Although the implementation of such a programme was not appropriate in the AOUG of THMG three decades ago, it should be considered for implementation now. A national survey should be carried out to estimate the current prevalence of anaemia among pregnant women in Sri Lanka. 


\section{References}

1. Lopez A, Cacoub P, Macdougall IC, Peyrin-Biroulet L. Iron deficiency anaemia- Seminar. The Lancet. 2015; 387(10021): 907-916.

2. World Health Organization. Worldwide prevalence of anaemia 1993 - 2005. WHO Global Database on Anaemia. de Benoist B, Mclean E, Egli I, Cogswell M Eds. Geneva, World Health Organization, 2008 (NLM: WH 155 ).

3. Department of Census and Statistics Sri Lanka. Prevalence of Anaemia among Children and Women. Demographic and Health Survey 2006/7, Health Sector Development Project, Ministry of Healthcare and Nutrition: Colombo, 2009.

4. Jayatissa R, Hossaine SMM. Nutrition and food security assessment in Sri Lanka 2009. Medical Research Institute, Sri Lanka, 2010

5. Fernandopulle PS. Prevalence of anaemia and some risk factors in pregnant women in DDHS area Dankotuwa, Dissertation for MD in Community Medicine. Postgraduate Institute of Medicine, University of Colombo, 1999 .

6. Sivaganesh S, Senarath U. Prevalence of antenatal risk conditions among women in an underserved district of Northern Sri Lanka. Ceylon Med J. 2009; 54: 110-114.

7. Chathurani U, Dharshika I, Galgamuwa D, Wickramasinghe N, Agampodi T, Agampodi S. Anaemia in pregnancy in the district of Anuradhapura, Sri Lanka - need for updating prevalence data and screening strategies. Ceylon MedJ. 2012; 57: 101-106.

8. Goonewardene M, Shehata M, Hamad A. Anaemia in pregnancy. Best Pract Res Clin Obstet \& Gynaecol. 2012; 26(1): 3-24

9. World Health Organization. Vitamin and Mineral Nutrition Information System. Haemoglobin concentrations for the diagnosis of anaemia and assessment of severity. Geneva: WHO 2011 (WHO/NMH/NHD/MNM/11.1).

10. Abbassi - Ghanavati M, Greer LG \& Cunningham FG. Pregnancy and laboratory studies: A reference table for clinicians. Obstet Gynaecol. 2009; 114(6): 1326-1331.

11. Palihawadana TS, Goonewardene IMR, Motha MBC, Williams HAS. Iron Deficiency Anaemia in Pregnancy: Diagnosis, Prevention and Treatment. Sri Lanka Journal of Obstetrics and Gynaecology. 2014; 36(3): 61-65.
12. Goonewardene. M. The Detection of Iron Deficiency in Pregnancy. Galle Medical Journal. 2001;3(1): 2-7.

13. Ray JG, Davidson AJF, Berger H, Dayan N, Park AL. Haemoglobin levels in pregnancy and severe maternal morbidity: population based cohort study. BJOG. 2020. DOI: 10.1111/1471-0528.1626.

14. Mwangi MN, Prentice AM, Verhoef H. Safety and benefits of antenatal oral iron supplementation in low-income countries: a review. British Journal of Haematology. 2017. bjh.14584.

15. World Health Organization, Regional Office for SouthEast Asia. Malaria-free Sri Lanka. ISBN 978-92-9022542-3 (NLM classification: WC 765). WHO SEARO, India 2016.

16. Peña-Rosas JP, De-Regil LM, Garcia-Casal MN, Dowswell T. Daily oral iron supplementation during pregnancy. Cochrane Database Syst Rev. 2015 Jul 22; (7): CD004736. DOI: 10.1002/14651858.CD004736.pub5.

17. Berger J, Dillon JC. Control of iron deficiency in developing countries. Sante. 2002; 12(1):22-30.

18. Goonewardene M. Is intermittent oral iron supplementation in pregnancy appropriate for South Asian Countries? Obstetrics and Gynaecology Communications. 2000; 2(4): 14-16.

19. Goonewardene M. Do all pregnant women need iron? Sri Lanka Journal of Obstetrics and Gynaecology. 1999; 21: $2-5$

20. Goonewardene M. Antenatal oral iron supplementation: why and how? Galle Medical Journal. 1997; 1: 11-14.

21. World Health Organization. Guideline: Daily iron and folic acid supplementation in adult women and adolescent girls. Geneva, World Health Organization, 2016

22. World Health Organization. Guideline: Intermittent iron and folic acid supplementation in menstruating women. Geneva, World Health Organization, 2011.

23. World Health Organization. Guideline: Intermittent iron and folic acid supplementation in non-anaemic pregnant women. Geneva, World Health Organization, 2012.

24. Goonewardene M, Seekkuge J, Liyanage C. Iron stores and its correlation to haemoglobin levels in pregnant women attending an antenatal clinic. Ceylon Medical Journal. 1995; 40: 67-69. 
25. Diluk Senadheera, Malik Goonewardene, Iresha Mampitiya. Anaemia and iron deficiency in pregnant women attending an antenatal clinic in a Teaching Hospital in Southern Sri Lanka. Ceylon Medical Journal. 2017; 62(3); 175-183.

26. Goonewardene IMR, Liyanage C. Efficacy of the current antenatal oral iron supplementation programme. Sri Lanka Journal of Obstetrics \& Gynaecology. 1996; 18: 14-17.

27. Goonewardene IMR, Fernando RJ, Liyanage C, Somasiri KG. The agreement between haematological indices obtained by different methods. Galle Medical Journal. 2000; 2(1): 13-17.

28. Diluk Senadheera, Malik Goonewardene. The agreement between Haematological indices obtained from two laboratories. Sri Lanka Journal of Obstetrics and Gynaecology, 2017;39(2): 23-26.

29. Goonewardene IMR, Fernando RJ, Liyanage C, Somasiri KG. The validity of commonly used haematological indices in the detection of iron deficiency in pregnancy. Ceylon Journal of Medical Science. 2001; 44(iv): 29-34.
30. Goonewardene IMR, Liyanage C. Antenatal iron supplementation; once a week only. Sri Lanka Journal of Obstetrics and Gynaecology. 1996; 18: 18-21.

31. Goonewardene IMR, Liyanage C. Antenatal iron supplementation; only thrice a week. Ceylon Journal of Medical Science. 1996; 39(1): 41-47.

32. Goonewardene $M$, Liyanage $C$, Fernando RJ. Intermittent oral iron supplementation during pregnancy. Ceylon Medical Journal. 2001; 46(iv): 132- 135.

33. Goonewardene IMR, Senadheera DI. Randomized control trial comparing effectiveness of weekly versus daily antenatal oral iron supplementation in preventing anaemia during pregnancy. The Journal of Obstetrics and Gynaecology Research 2018; 44(3): 417-424. 